CLAWAR 2018: 21st International Conference on Climbing and Walking Robots and the Support Technologies for Mobile Machines, Panama City, Panama, 10-12 September 2018

\title{
STABLE WALKING DYNAMICS IN A SEMI-PASSIVE BIPED
}

\author{
CARLOS F. RODRIGUEZ HERRERA \\ Department of Mechanical Engineering, Universidad de Los Andes, Carrera 1 Este No. \\ $19 A$ - 40 Bogotá, DC, Colombia \\ JUAN CAMILO MARTINEZ FRANCO \\ Department of Mechanical Engineering, Universidad de Los Andes, Carrera 1 Este No. \\ $19 A-40$ Bogotá, DC, Colombia \\ SEBASTIAN MURILLO HERRERA \\ Department of Mechanical Engineering, Universidad de Los Andes, Carrera 1 Este No. \\ 19A - 40 Bogotá, DC, Colombia
}

\begin{abstract}
Semi-passive biped robots have been proposed to achieve efficient and robust bipedal locomotion, based on their natural dynamics. In this work, a compass biped with arc shape feet, actuated by a rocker mechanism on the hip is presented and studied. Initially, the effects of the variation of the excitation frequency and amplitude on the stability of the walking cycle are determined. Both, simulation results and experimental tests, suggest that, for a given range of excitation frequencies, the robot exhibits attractive equilibrium as well as entrainment. In addition, strategies for startup, turning and stopping were explored, obtaining promising results. This work supports the possibility of developing controllable, energy efficient, semi-passive, low cost bipeds.
\end{abstract}

\section{Introduction}

Bipedal robots are of interest because of their similarity to humans, but compared with robots on wheels, or even robots with four or more legs, bipedal robots seem to have few practical uses nowadays. Some of their drawbacks include low efficiencies and difficulty to control. However, bipedal robots have numerous potential applications in several fields: their locomotion can help understand human gait; traverse through irregular terrain, and they are the base for developing humanoid robots that can operate infrastructure designed for people.

Regarding energy efficiency, it has been suggested that by taking advantage of the natural dynamics of a system, it is possible to produce locomotion with a low cost of transport. Such ideas where demonstrated by the passive dynamic walker, developed by McGreer [1]. Passive dynamic walkers do not have any actuation and depend on the conversion of potential energy to maintain a stable 
walking cycle, as friction and partially elastic collisions drain energy from the system. In this paper, we study a simple semi-passive biped developed as a passive dynamic walker equipped with a servo-actuated rocker mechanism on the hip, following the proposals of Collins et al. [2], in which the gravitational power is substituted by a simple actuation. The biped, initially developed by Botero et al. [3], was designed after a reviewing several passive compass gait robots with arc-shaped feet.

The mathematical model for passive dynamic walking is non-linear in nature, more so if actuation is to be added. Instead of addressing this non-linear model, we propose a series of dynamic simulations to gain insight of the robot's behavior. The software used for both modelling and simulation is Autodesk Inventor, that includes a variable-step dynamic simulation environment with a solver using a five-order Runge-Kutta integration scheme [4]. Different simulations were run to evaluate the stability and estimate the degree to which the robot can be controlled. A prototype was constructed and tested to validate the simulations.

The paper is presented as follows: section 2 contains the physical description of the robot as well as the assumptions made during modelling. Simulation parameters and trajectories are described on section 3. The results of the simulations and experiments with the prototype are presented and discussed on section 4. Conclusions and future work can be found on section 5 .

\section{Semi-passive biped description}

The semi-passive biped (SPB) developed for this study is based on a knee-less passive dynamic walker: a compass biped with arc-shape feet. This is one of the simplest passive walkers since it is statically stable and only has two rotational joints in the hips. The actuation power is a rocker mechanism attached to the hip of the passive walker. Roughly, this mechanism provides swinging motion in a coronal plane allowing the biped to walk with a controlled gait on a flat surface. The proposed SPB results in a low cost prototype, with potential to study dynamic characteristics and control of the gait in robots or humans.

\subsection{Passive walker}

The base of the SPB is a passive walker (figure 1). It is a biped without actuation consisting of a $500 \mathrm{~mm}$ long steel shaft (hip) and two $400 \mathrm{~mm}$ long aluminum square profile (legs) ending in a spherical section wooden assembly (feet) with a radius of $120 \mathrm{~mm}$. 
This walker has a weight of $950 \mathrm{~g}$. It can maintain a repeating gait in ramps with slope angles ranging from 1.5 to 8 degrees, exhibiting a synchronized lateral swinging which allows each leg to oscillate alternately to produce the gait.
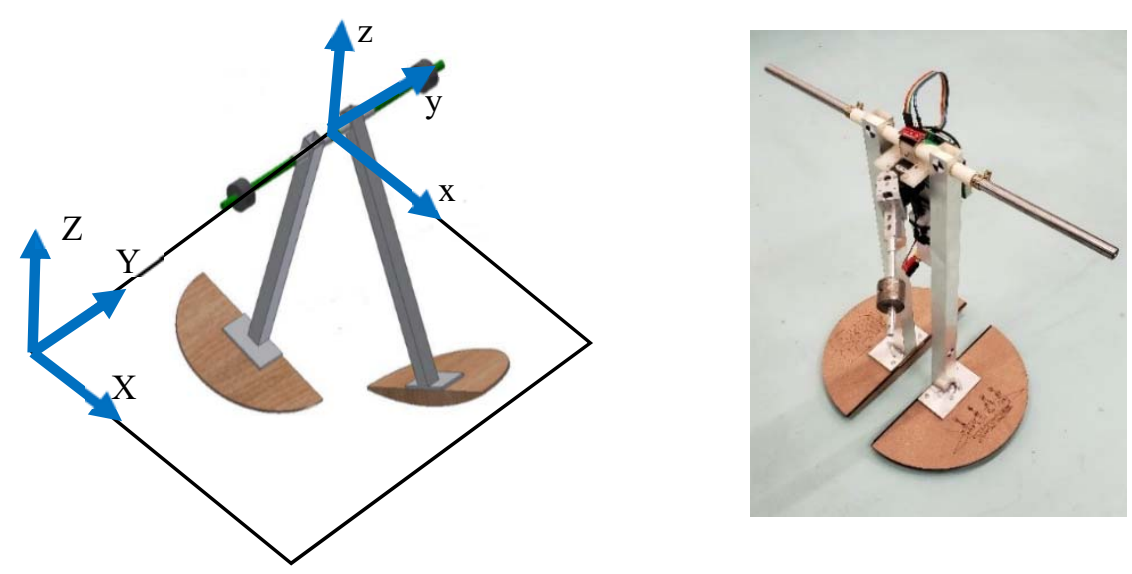

Figure 1. a) 3D Walker model. B)SPB

\subsection{Actuation mechanism}

Once a passive walker has an initial impulse, it needs additional energy to move on a flat surface. Enough power must be provided to produce the walking motion, while compensate the energy dissipated because of friction and impact damping. In this SPB, the actuation is a rocker mechanism on the hip, which generates the following effects: preserves its lateral swinging (roll), produces inclination in the forward direction (pitch), and generates inertial moment in coronal plane (yaw).

Figure 1 shows the SPB with the actuation mechanism. The rocker consists of an $80 \mathrm{~g}$ mass attached to a $150 \mathrm{~mm}$ rod, coupled to a Dynamixel AX-12A servomotor. The mass rotates around the shaft of the motor, in the plane $x-y$ (figure 1). This motor can deliver a rated torque of $1.5 \mathrm{Nm}$ at $59 \mathrm{rpm}$, enough to produce the required operation oscillation frequency. A Raspberry Pi 3 controls the servomotor.

Garcia et al. [5] proposed a system of equations that describe the walker without actuation. While it is possible to extend this methodology to formulate a similar system for the SPB, it would result in a non-linear model, requiring some mathematical manipulation to become useful for control purposes. Instead, we propose to explore the behavior of the SPB via simulation and testing on a prototype, to gain some insight of its control possibilities. 


\section{Simulation parameters}

The SPB was modelled in Autodesk Inventor ${ }^{\circledR}$ using the geometry and mass properties previously described. The settings for the motion of the rocker mechanism are the following: amplitude from 40 to 60 degrees, frequency between 0.8 and $1.2 \mathrm{~Hz}$, and offset from -15 to 15 degrees. These testing conditions were selected after some experimentation on the passive walker prototype and some initial simulations.

\subsection{Cyclic stable gait and entrainment}

A system can be forced to oscillate to a given frequency with little energy input if the selected frequency is close to the system's natural frequency. This phenomenon is called entrainment and it is necessary for the control of the SPB.

Since the gait cycle frequency of the passive walker prototype in a ramp with a slope of 1.5 degrees was of $1.05 \mathrm{~Hz}$, the rocker mechanism was actuated for the first set of simulations following a sinewave trajectory with frequencies between $0.8 \mathrm{~Hz}$ and $1.2 \mathrm{~Hz}$.

\subsection{Contact and damping}

Impact is modelled in the Autodesk Inventor dynamic simulation environment as a series of elastic collisions with a damping coefficient. Marin [6] conducted an experimental estimation of these parameters for the passive walker prototype on a wooden ramp. For the simulations, we used the values obtained by Marin [6] (rigidity $\mathrm{K}=10 \mathrm{~N} / \mathrm{mm}$, dampening coefficient $\mathrm{C}=5.142 \mathrm{Ns} / \mathrm{mm}$ and coefficient of friction $\mu=0.3572$ ), as nominal values with variations of $5 \%$ and $10 \%$, in order to consider the influence of possible variations in a real level-ground.

\section{Simulation and experimental results}

\subsection{Cyclic stable gait}

To determine the operational conditions for a cyclic stable gait of the SPB, the conditions of simulation were simplified setting the offset to zero. Input frequency varied from $0.8 \mathrm{~Hz}$ to $1.2 \mathrm{~Hz}$ in steps of $0.1 \mathrm{~Hz}$ and amplitude varied between $40^{\circ}$ and $60^{\circ}$ in steps of $5^{\circ}$. The initial condition was set to a roll angle of $8^{\circ}$ and zero initial velocity.

The SPB shows a cyclic stable gait for a range of input frequencies depending on the contact conditions (figure 2). As was expected, the gait is stable for frequencies up to the natural frequency of the UBR $(1.05 \mathrm{~Hz})$. 


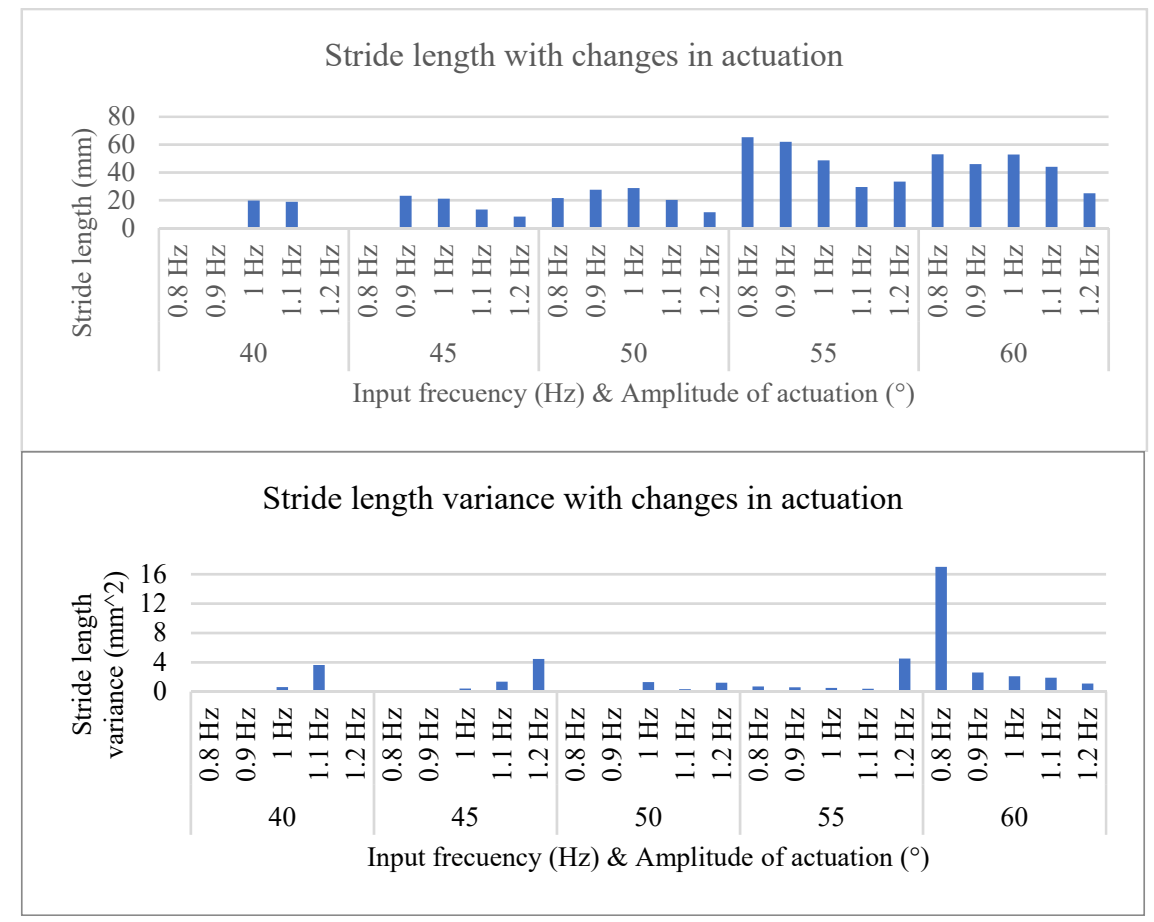

Figure 2. Cyclic stable gait

Figure 3 shows the horizontal view of the evolution of the trajectory of the center of mass of the hip for a set of input conditions (frequency of $1 \mathrm{~Hz}$, zero offset and proposed contact conditions).

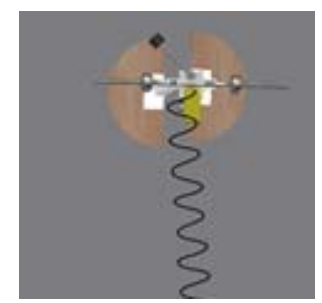

Figure 3. Trajectory of the center of mass of the hip.

The operating conditions were defined as $\mathrm{K}=10 \mathrm{~N} / \mathrm{mm}, \mathrm{C}=5.172 \mathrm{Ns} / \mathrm{mm}$ and $\mu=0.3571$ with marginal variations. The interval of operating frequencies depends on the contact settings. Simulation results are shown on Figure 4. The marginal changes in $\mathrm{K}$ doesn't produce important effects in the gait cycle. 


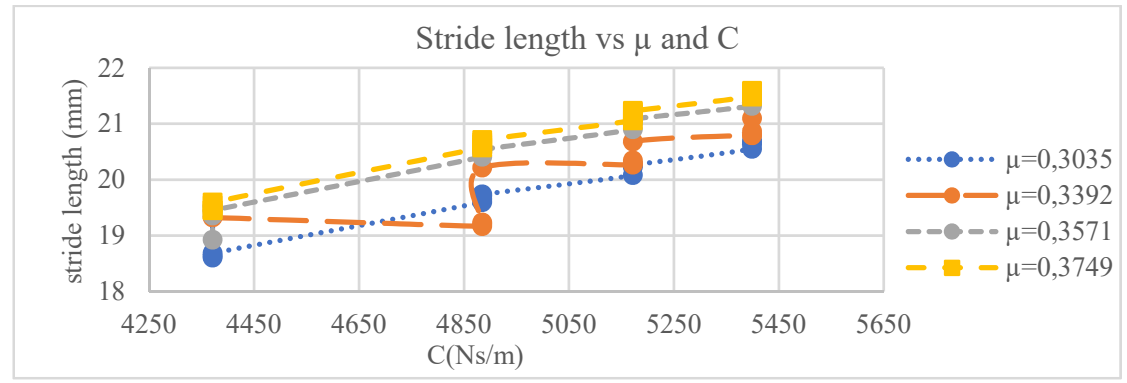

Figure 4. Marginal changes in contact conditions

In all cases, frequency entrainment was observed, to various degrees, although many of them do not produce gait. If the rocker is actuated in phase with the roll oscillation, the later will stop to a halt. This effect of the phase of the input with respect to the roll angle of the SPB is shown in Figure 5.

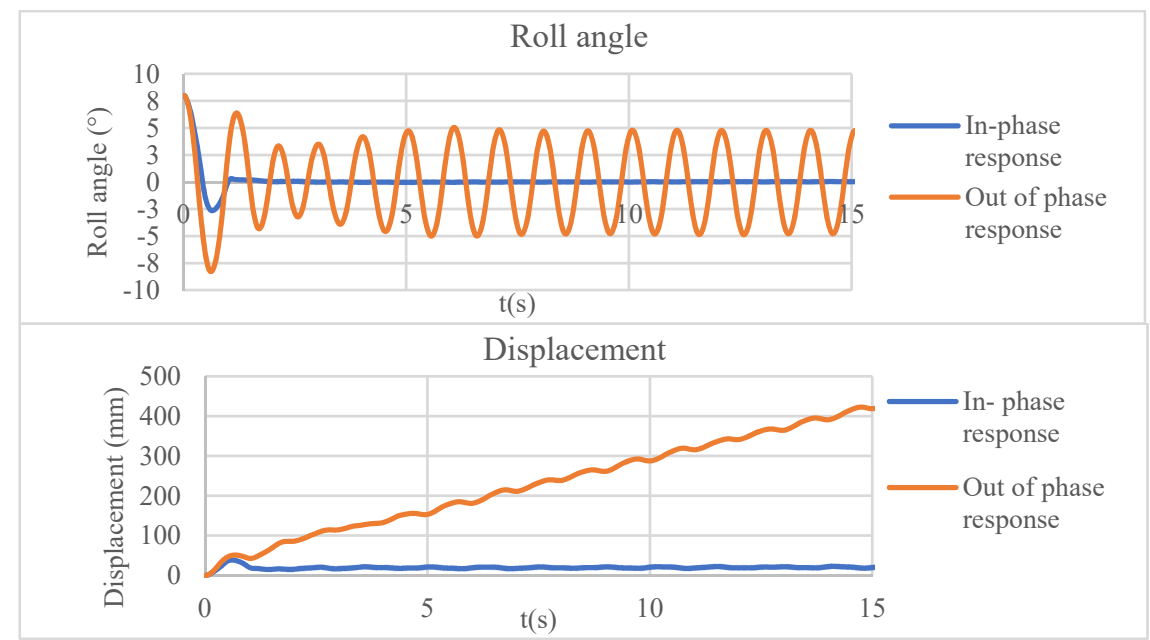

Figure 5. Rocker oscillation phase response in phase and out of phase (half a period).

\subsection{Starting and stopping}

Simply rocking the mass in the frequency that produces the best results $(1 \mathrm{~Hz})$ will produce an entrainment in approximately the same phase, which, as it was explained above, won't result in a gait cycle.

In this regard, a "startup trajectory" was proposed, where the rocker is actuated too rapidly for the SPB to respond with entrainment. Instead, the SPB is slowly forced out of its stable stationary state, until conditions approximating the 
ones presented in the previous section are reached. In the same way, actuating the walker in-phase will cause it to stop walking.

\subsection{Turning}

Up to this point, the input function of the rocker mechanism had no offset around the $\mathrm{x}$-axis of the SPB. This condition produces the SPB gait to be straightforward. When the input function has an offset, the SPB gait follows a curved trajectory. Table 1 shows the trajectory of the center of mass of the SPB when the amplitude of the input function is displaced 15 degrees towards the right (a) and to the left (b).

Table 1. SPB turning radius response to amplitude displacement, measured on the transverse plane.

\begin{tabular}{cc}
\hline Offset angle $\left(^{\circ}\right)$ & Turn radius $(\mathbf{m})$ \\
\hline 0 & - \\
2,5 & 11,9 \\
5 & 7,8 \\
7,5 & 12,4 \\
10 & 2,5 \\
12,5 & 2,0 \\
15 & 1,5 \\
\hline
\end{tabular}

The amplitude displacement results in a change of direction as expected, albeit at the cost of a relatively unstable gait. This opens the possibility partial control of one additional degree of freedom with the same actuator.

\subsection{Experimental results.}

The most reasonable dynamic parameters, found in simulation work, where replicated with the physical SPB. The prototype exhibited a cyclic gait very similar to the one observed in simulation. Acceleration and orientation data were collected with an inertial measurement unit (IMU), with the acceleration on the SBP's $x$ axis integrated to observe the travelled distance, as seen on figure 6 .

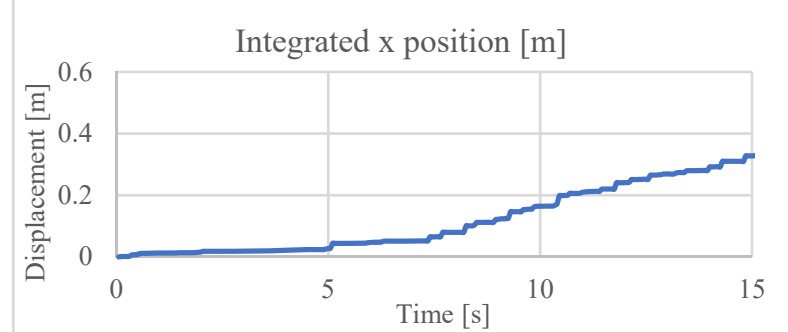

Figure 6. Position response on the physical SBP during experimental tests at $1 \mathrm{~Hz}$ and $45^{\circ}$ of amplitude in actuation 


\section{Conclusions}

This paper presents an underactuated biped robot based on a passive dynamic walker with a simple hip rocker mechanism. The proposed system was studied using dynamic simulation to establish general parameters of the actuation conditions for controlling its gait. Three gaits were studied: stable cyclic gait, starting and stopping, and turning.

Simulation and experimental results shows that it is possible to achieve entrainment between certain input frequencies and the gait frequency, when the input is half a period out of phase form the gait. A strategy for stopping the gait is also derived from the behavior of the gait with an input in phase with the gait. An excitation with a frequency higher than the natural frequency of the system can provide a starting condition. Finally, turning can be achieved by adding offset to the input signal.

This work shows a promising development direction for simple biped robots, especially considering the low cost of the prototype. Further simulation and experimental work could be useful to understand the effects of external conditions on the walking cycle of the robot. However, after this exploratory phase, mathematical modelling of the system is also necessary.

\section{References}

1. T. McGreer, Passive Dynamic Walking, The International Journal of Robotics Research, Vol. 9, Issue 2, pp. 62-82 (1990).

2. S. Collins, A. Ruina, R. Tedrake, M. Wisse, Efficient Bipedal Robots Based on Passive-Dynamic Walkers, Science, Vol 307, pp. 1082-1085 (2005).

3. C. Botero, J. Montoya, N. Sánchez, F. Vergara, Development of a passive dynamic walker prototype, Intermediate project report, Universidad de los Andes (2012).

4. W. Younis, Up and Running with Autodesk Inventor Simulation 2011, Elsevier, $2^{\text {nd }}$ ed., Burlington (2010).

5. M. Garcia, A. Chatterjee, A. Ruina, M. Coleman, The Simplest Walking Model: Stability, Complexity, and Scaling, Journal of Biomechanical Engineering, Vol. 120, Issue 2, pp. 281-288 (1998).

6. D. Marín, 3D Dynamic Modeling and Observer Design for a Passive Biped Walker, MSc. Thesis, Universidad de los Andes (2017). 